\title{
University of Florida Potato Variety Trials Spotlight: ‘Harley Blackwell”
}

\author{
Mario H. M. L. Andrade, Rodrick Z. Mwatuwa, Christian T. Christensen, Pam Solano, Kathleen \\ G. Haynes, and Lincoln Zotarelli²
}

\section{Introduction}

There are several potato varieties available in the market today. Most of them have been bred or developed in production regions other than Florida. The University of Florida Potato Variety Evaluation Program screens new germplasm from public and private breeding programs and identifies the most promising cultivars for commercial potential considering broad adaptability to Florida climate and conditions and market purpose: processing, freshmarket and specialty-type varieties. Over the years, the UF/ IFAS Potato Variety Program has become an important reference to vegetable growers, seed producers, processors, crop insurance agencies, and brokers looking for alternative potato varieties to explore different markets, improved characteristics, and yield. This UF/Potato Variety Trials Spotlight presents a summary of the field evaluation of tuber yield and quality performance of the potato variety 'Harley Blackwell' cultivated in Florida.

\section{General Comments}

'Harley Blackwell' is a potato variety that is commonly grown for the potato chip market. It was selected from the progeny of a cross between B0155-24 and B9935-8 and tested under the pedigree B0564-8. It was released and named jointly by the Agricultural Research Service, the United States Department of Agriculture, the Agricultural Research Service of North Carolina, the Agricultural Experiment Stations of Virginia, New Jersey, Pennsylvania, Florida, and New York, and the Maine Agricultural and Forest Experiment Station in 2000. Tuber production and quality results provided in this spotlight are summarized from various variety trials conducted at the UF/IFAS Hastings Agricultural Extension Center between 1998 and 2019.

\section{General Characteristics}

'Harley Blackwell' tubers are mostly round with a white flesh color (Figure 1). According to Florida's rating codes for potato tuber characteristics (Table 1), the tubers have a good appearance with buff skin color, slightly netted skin texture, and intermediate to shallow eye depth (Table 3). 'Harley Blackwell' demonstrates high yield potential under Florida production conditions (Tables 2 and 3). On average, marketable yield is $265 \mathrm{cwt} /$ acre with $83 \%$ of the tubers produced found between A1 and A3 tuber size classification and low incidence of internal defects like internal heat necrosis. The variety has a medium to high specific gravity of 1.074 (Table 2).

1. This document is HS1298, one of a series of the Horticultural Sciences Department, UF/IFAS Extension. Original publication date May 2017. Revised February 2021. Visit the EDIS website at https://edis.ifas.ufl.edu.

2. Mario H. M. L. Andrade, research scholar; Rodrick Z. Mwatuwa, research assistant; Christian T. Christensen, regional specialized agent II, director, UF/ IFAS Hastings Agricultural Extension Center; Pam Solano, biological scientist; Kathleen G. Haynes, Genetic Improvement of Fruits and Vegetables Laboratory, USDA-ARS, Beltsville, MD; and Lincoln Zotarelli, associate professor; Horticultural Sciences Department, UF/IFAS Extension, Gainesville, FL 32611.

The Institute of Food and Agricultural Sciences (IFAS) is an Equal Opportunity Institution authorized to provide research, educational information and other services

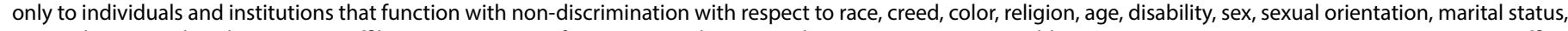

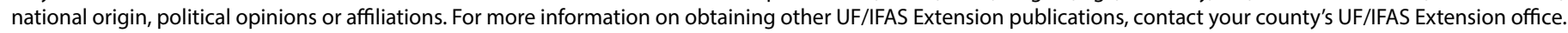
U.S. Department of Agriculture, UF/IFAS Extension Service, University of Florida, IFAS, Florida A \& M University Cooperative Extension Program, and Boards of County Commissioners Cooperating. Nick T. Place, dean for UF/IFAS Extension. 


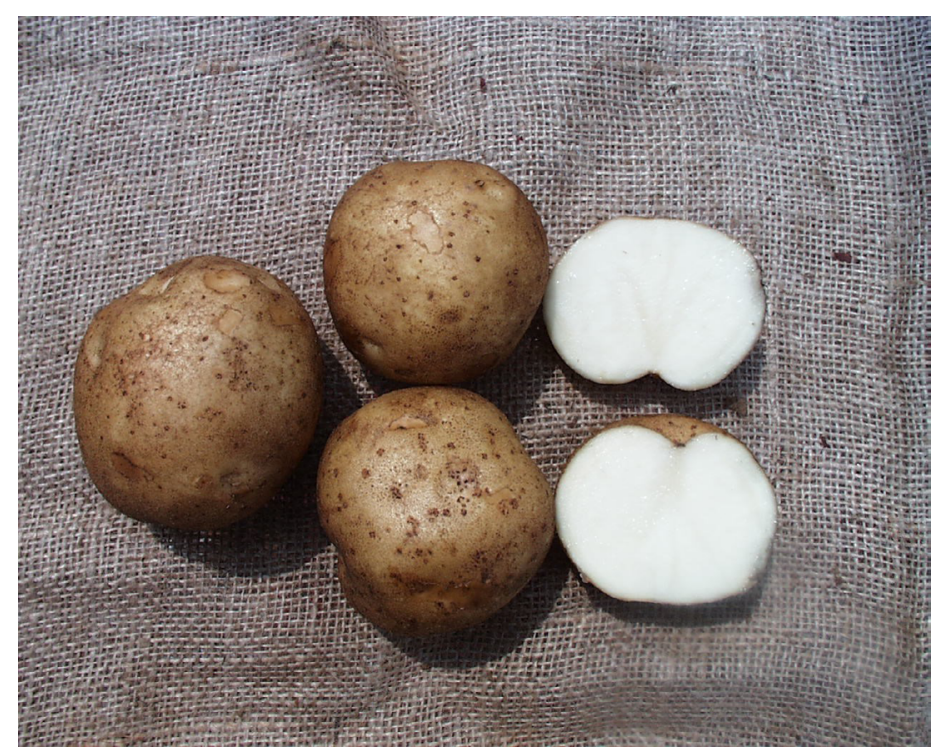

Figure 1. Typical tuber and internal flesh color of 'Harley Blackwell' potato variety.

Credits: Kathleen Haynes, UF/IFAS

\section{Diseases}

'Harley Blackwell' is resistant to race A of the golden nematode (Globodera rostochiensis). It is susceptible to Verticillium wilt (Verticillium albo-atrum and Verticillium dahliae) and late blight (Phytophthora infestans). It is moderately susceptible to early blight (Alternaria solani), with intermediate resistance to common scab (Streptomyces scabies), and some tolerance to powdery scab (Spongospora subterranea f. sp. subterranea). The standard UF/IFAS Extension recommended disease and weed control program described under Potato Production (Chapter 14 of the Vegetable Production Handbook for Florida, http://edis.ifas. ufl.edu/cv131) should be followed.

\section{Season Length and Growth}

'Harley Blackwell' is an early- to medium-maturing variety. Season length was 100 days on average from planting to harvest. This depended on weather conditions during the growing season. Late in the season, tuber size should be closely monitored to harvest tubers with marketable size. Soil moisture should be managed late in the season to avoid high soil moisture conditions that cause enlarged lenticels, which are sites of entry for decay organisms.

\section{Fertilization}

UF/IFAS trial plots are normally fertilized with 200 to 230 $\mathrm{lb} / \mathrm{ac}$ of $\mathrm{N}$. The first application of $100 \mathrm{lb} / \mathrm{ac}$ of $\mathrm{N}$ (granular) is typically incorporated in the bed 2 to 5 days prior to planting, followed by one or two side-dress fertilizer applications at emergence and/or at tuber initiation. Phosphorus and potassium applications follow the UF/IFAS guidelines described in Liu et al. (2020) and normally range between 45 to $100 \mathrm{lb} / \mathrm{ac}$ of $\mathrm{P}_{2} \mathrm{O}_{5}$ and 170 to $235 \mathrm{lb} / \mathrm{ac}$ of $\mathrm{K}_{2} \mathrm{O}$.

\section{Planting}

A seed piece of $2^{1 / 2}$ to $3 \mathrm{oz}$ is recommended for planting. This variety should be planted with 40 inches between rows and 8 inches between plants, at 3 to 4 inches deep. A seed rate of 2,000 to 3,000 lb/acre of seed is expected.

\section{Other Information}

For additional information on cultivation and weed and disease management, see the Potato Production chapter of the Vegetable Production Handbook, available at http://edis. ifas.ufl.edu/cv131.

\section{References}

Hutchinson, C. M., J. M. White, D. M. Gergela, P. A. Solano, K. G. Haynes, R. Wenrich, and C. S. Lippi. 2003. "Performance of chip processing potato varieties in northeastern Florida." HortTechnology 13 (4): 706-711. https://doi. org/10.21273/HORTTECH.13.4.0706

Liu, G., E. H. Simonne, K. T. Morgan, G. J. Hochmuth, S. Agehara, and R. Mylavarapu. 2020. Chapter 2. Fertilizer Management for Vegetable Production in Florida. Vegetable Production Handbook for Florida, 2020-2021 Edition. CV296. Gainesville: University of Florida Institute of Food and Agricultural Sciences. http://edis.ifas.ufl.edu/cv296

Navarre, R., and M. Pavek. 2014. The Potato: Botany, Production and Uses. CABI. https://doi. org/10.1079/9781780642802.0000

Sisson, J. A., and G. A. Porter. 2002. "Performance evaluations of potato clones and varieties in the northeastern states-1999." Maine Agr. For. Expt. Sta., Misc. Publ. 751.

USDA-ARS. 2016. "Naming and Release Of the 'Harley Blackwell' Potato Variety." https://www.ars. usda.gov/Services/Docs.htm?docid=19468. Accessed 2 February 2021.

Zotarelli, L., P. J. Dittmar, P. D. Roberts, J. Desaeger, and B. Wells. 2020. Chapter 14. Potato Production. Vegetable Production Handbook for Florida, 2020-2021 Edition. HS733. Gainesville: University of Florida Institute of Food and Agricultural Sciences. http://edis.ifas.ufl.edu/cv131 
Table 1. Florida rating codes for potato vine maturity and tuber characteristics.

\begin{tabular}{|c|c|c|c|c|c|c|c|}
\hline \multicolumn{5}{|c|}{ Tuber Characteristics $^{1}$} & \multirow[b]{2}{*}{ Tuber Shape } & \multirow[b]{2}{*}{$\begin{array}{c}\text { Eye } \\
\text { Depth }\end{array}$} & \multirow[b]{2}{*}{$\begin{array}{c}\text { Overall Tuber } \\
\text { Appearance }\end{array}$} \\
\hline Rating Code & Vine Maturity & $\begin{array}{l}\text { Internal Flesh } \\
\text { Color }\end{array}$ & Skin Color & Skin Texture & & & \\
\hline 1 & dead & white & purple & partial russet & round & very deep & very poor \\
\hline 2 & +- & cream & red & heavy russet & mostly round & -- & -- \\
\hline 3 & $\begin{array}{l}\text { yellow and } \\
\text { dying }\end{array}$ & light yellow & pink & $\begin{array}{c}\text { moderate } \\
\text { russet }\end{array}$ & $\begin{array}{l}\text { round to } \\
\text { oblong }\end{array}$ & deep & poor \\
\hline 4 & +- & medium yellow & dark brown & light russet & mostly oblong & -- & -- \\
\hline 5 & $\begin{array}{c}\text { moderately } \\
\text { senesced }\end{array}$ & dark yellow & brown & netted & oblong & intermediate & fair \\
\hline 6 & +- & pink & $\tan$ & slightly netted & oblong to long & -- & -- \\
\hline 7 & $\begin{array}{l}\text { starting to } \\
\text { senesce }\end{array}$ & red & buff & $\begin{array}{l}\text { moderately } \\
\text { smooth }\end{array}$ & mostly long & shallow & good \\
\hline 8 & +- & blue & white & smooth & long & -- & -- \\
\hline 9 & $\begin{array}{l}\text { green and } \\
\text { vigorous }\end{array}$ & purple & cream & very smooth & cylindrical & very shallow & excellent \\
\hline
\end{tabular}

Table 2. Summary of production statistics and specific gravity of 'Harley Blackwell' grown at the UF/IFAS Hastings Agricultural Extension Center, Hastings, FL from 1998 to 2019, excluding 2001.

\begin{tabular}{|c|c|c|c|c|c|c|c|c|c|c|c|c|c|}
\hline \multirow[t]{2}{*}{ Year } & \multirow{2}{*}{$\begin{array}{c}\text { Total } \\
\text { Yield } \\
\text { (cwt/ac) }\end{array}$} & \multirow{2}{*}{$\begin{array}{c}\text { Marketable } \\
\text { Yield (cwt/ } \\
\text { ac) }{ }^{1}\end{array}$} & \multirow{2}{*}{$\begin{array}{l}\% \text { of } \\
\text { STD }\end{array}$} & \multirow[t]{2}{*}{ Standard } & \multicolumn{6}{|c|}{ Size Class (Distribution by Class \%) } & \multicolumn{2}{|c|}{ Range \% } & \multirow{2}{*}{$\begin{array}{l}\text { Specific } \\
\text { Gravity }\end{array}$} \\
\hline & & & & & C & B & A1 & A2 & A3 & A4 & $\begin{array}{c}\text { A1 to } \\
\text { A3 }\end{array}$ & $\begin{array}{c}\% \\
\text { Culls }\end{array}$ & \\
\hline 1998 & 390 & 307 & 84 & Atlantic & 0 & $22^{*}$ & 46 & 25 & 7 & 0 & 79 & 14 & 1.072 \\
\hline 1999 & 384 & 342 & 97 & Atlantic & 0 & $11^{*}$ & 79 & 10 & 0 & 0 & 89 & 5 & 1.065 \\
\hline 2000 & 290 & 254 & 73 & Atlantic & 0 & $13^{*}$ & 39 & 30 & 18 & 0 & 88 & 8 & 1.073 \\
\hline 2002 & 256 & 235 & 83 & Atlantic & 0 & $6^{*}$ & 52 & 35 & 7 & 0 & 94 & 3 & 1.076 \\
\hline 2003 & 416 & 344 & 86 & Atlantic & 0 & $14^{*}$ & 39 & 30 & 16 & 1 & 85 & 3 & 1.075 \\
\hline 2004 & 347 & 282 & 86 & Atlantic & 11 & 8 & 51 & 25 & 5 & 0 & 81 & 1 & 1.087 \\
\hline 2005 & 339 & 290 & 102 & Atlantic & 1 & 13 & 69 & 15 & 2 & 0 & 86 & 0 & 1.083 \\
\hline 2006 & 401 & 333 & 89 & Atlantic & 1 & 14 & 74 & 10 & 1 & 0 & 84 & 1 & 1.087 \\
\hline 2007 & 359 & 303 & 82 & Atlantic & 2 & 12 & 65 & 16 & 5 & 0 & 86 & 2 & 1.075 \\
\hline 2008 & 341 & 279 & 98 & Atlantic & 3 & 14 & 57 & 17 & 11 & 0 & 84 & 3 & 1.079 \\
\hline 2009 & 356 & 288 & 105 & Atlantic & 2 & 11 & 67 & 12 & 8 & 0 & 87 & 8 & 1.066 \\
\hline 2010 & 367 & 253 & 93 & Atlantic & 4 & 24 & 65 & 5 & 2 & 0 & 72 & 5 & 1.072 \\
\hline 2011 & 331 & 278 & 106 & Atlantic & 3 & 11 & 63 & 17 & 6 & 0 & 86 & 3 & 1.074 \\
\hline 2012 & 363 & 300 & 70 & Atlantic & 2 & 9 & 57 & 16 & 15 & 0 & 88 & 7 & 1.074 \\
\hline 2013 & 314 & 282 & 110 & Atlantic & 2 & 7 & 59 & 18 & 14 & 0 & 92 & 2 & 1.071 \\
\hline 2014 & 295 & 192 & 85 & Atlantic & 5 & 25 & 60 & 6 & 4 & 0 & 70 & 7 & 1.068 \\
\hline 2015 & 279 & 201 & 79 & Atlantic & 3 & 15 & 64 & 10 & 8 & 0 & 82 & 13 & 1.067 \\
\hline 2016 & 263 & 167 & 61 & Atlantic & 9 & 23 & 56 & 6 & 6 & 0 & 67 & 6 & 1.071 \\
\hline 2017 & 229 & 144 & 70 & Atlantic & 8 & 18 & 65 & 6 & 3 & 0 & 74 & 16 & 1.075 \\
\hline 2018 & 311 & 247 & 107 & Atlantic & 5 & 13 & 68 & 8 & 7 & 0 & 82 & 4 & 1.076 \\
\hline 2019 & 312 & 249 & 75 & Atlantic & 5 & 8 & 28 & 52 & 3 & 0 & 87 & 9 & 1.077 \\
\hline Average & 331 & 265 & 88 & Atlantic & 3 & 14 & 58 & 18 & 7 & 0 & 83 & 6 & 1.074 \\
\hline \multicolumn{14}{|c|}{$\begin{array}{l}{ }^{1} \text { Marketable yield: Sum of size classes } A 1 \text { to } A 3 \text {. } \\
{ }^{2} \text { Size classes: } C=0.5 \text { to } 1.5 \text { inches, } B=1.5 \text { to } 17 / 8 \text { inches, } A 1=17 / 8 \text { to } 2.5 \text { inches, } A 2=2.5 \text { to } 3.25 \text { inches, } A 3=3.25 \text { to } 4 \text { inches, } A 4>4 \text { inches; } \\
\text { Size distribution by class: Class (wt)/(Total Yield [wt] - culls [wt]) n.a. = not available } \\
{ }^{*} \text { Classification }=<17 / 8 \text { inches ( } C \text { and B included in this classification) }\end{array}$} \\
\hline
\end{tabular}


Table 3. Vine maturity, tuber characteristics, and internal tuber defects of 'Harley Blackwell' potato variety grown at the UF/IFAS Hastings Agricultural Extension Center, Hastings, FL from 1998 to 2019, excluding 2001.

\begin{tabular}{|c|c|c|c|c|c|c|c|c|c|c|c|}
\hline \multirow[t]{2}{*}{ Year } & \multirow{2}{*}{$\begin{array}{c}\text { Vine } \\
\text { Maturity }\end{array}$} & \multicolumn{6}{|c|}{ Tuber Characteristics $^{1}$} & \multirow[b]{2}{*}{ HH } & \multicolumn{2}{|c|}{ Internal Defects² } & \multirow[b]{2}{*}{ IHN } \\
\hline & & $\begin{array}{c}\text { Internal } \\
\text { Flesh } \\
\text { Color }\end{array}$ & $\begin{array}{l}\text { Skin } \\
\text { Color }\end{array}$ & $\begin{array}{l}\text { Skin } \\
\text { Texture }\end{array}$ & $\begin{array}{l}\text { Tuber } \\
\text { Shape }\end{array}$ & $\begin{array}{c}\text { Eye } \\
\text { Depth }\end{array}$ & $\begin{array}{c}\text { Overall } \\
\text { Appearance }\end{array}$ & & BR & CRS & \\
\hline 1998 & 0 & 0 & 8 & 7 & 3 & 6 & 7 & 0 & 0 & 0 & 0 \\
\hline 1999 & 0 & 0 & 8 & 5 & 2 & 4 & 6 & 0 & 0 & 0 & 0 \\
\hline 2000 & 0 & 0 & 6 & 5 & 2 & 6 & 6 & 0 & 0 & 0 & 0 \\
\hline 2002 & 2 & 2 & 7 & 6 & 2 & 7 & 7 & 0 & 0 & 0 & 1 \\
\hline 2003 & 5 & 1 & 6 & 5 & 2 & 6 & 7 & 12 & 0 & 0 & 0 \\
\hline 2004 & 4 & 1 & 7 & 5 & 2 & 7 & 7 & 0 & 0 & 0 & 0 \\
\hline 2005 & 6 & 1 & 6 & 5 & 2 & 7 & 7 & 0 & 0 & 0 & 0 \\
\hline 2006 & * & 1 & 6 & 6 & 3 & 6 & 6 & 0 & 0 & 0 & 0 \\
\hline 2007 & 6 & 2 & 6 & 5 & 3 & 5 & 7 & 0 & 0 & 0 & 0 \\
\hline 2008 & 5 & 2 & 7 & 5 & 2 & 6 & 7 & 0 & 0 & 0 & 0 \\
\hline 2009 & 3 & 1 & 6 & 5 & 3 & 4 & 6 & 1 & 0 & 0 & 0 \\
\hline 2010 & 7 & 1 & 6 & 5 & 3 & 6 & 7 & 2 & 0 & 0 & 1 \\
\hline 2011 & 3 & 1 & 6 & 5 & 2 & 5 & 7 & 0 & 0 & 11 & 2 \\
\hline 2012 & 5 & 2 & 6 & 5 & 3 & 5 & 6 & 1 & 0 & 0 & 1 \\
\hline 2013 & 5 & 1 & 6 & 5 & 3 & 4 & 6 & 2 & 0 & 0 & 2 \\
\hline 2014 & 2 & 1 & 7 & 5 & 3 & 5 & 7 & 0 & 0 & 0 & 0 \\
\hline 2015 & 6 & 1 & 9 & 8 & 2 & 7 & 8 & 0 & 2 & 0 & 0 \\
\hline 2016 & 7 & 1 & 6 & 7 & 2 & 7 & 7 & 0 & 0 & 0 & 0 \\
\hline 2017 & 7 & 1 & 8 & 5 & 2 & 8 & 7 & 0 & 0 & 0 & 0 \\
\hline 2018 & 7 & 1 & 7 & 7 & 2 & 8 & 8 & 0 & 0 & 0 & 0 \\
\hline 2019 & 6 & 1 & 6 & 7 & 2 & 7 & 7 & 0 & 0 & 0 & 0 \\
\hline Average & 4 & 1 & 7 & 6 & 2 & 6 & 7 & 1 & 0 & 1 & 0 \\
\hline
\end{tabular}

